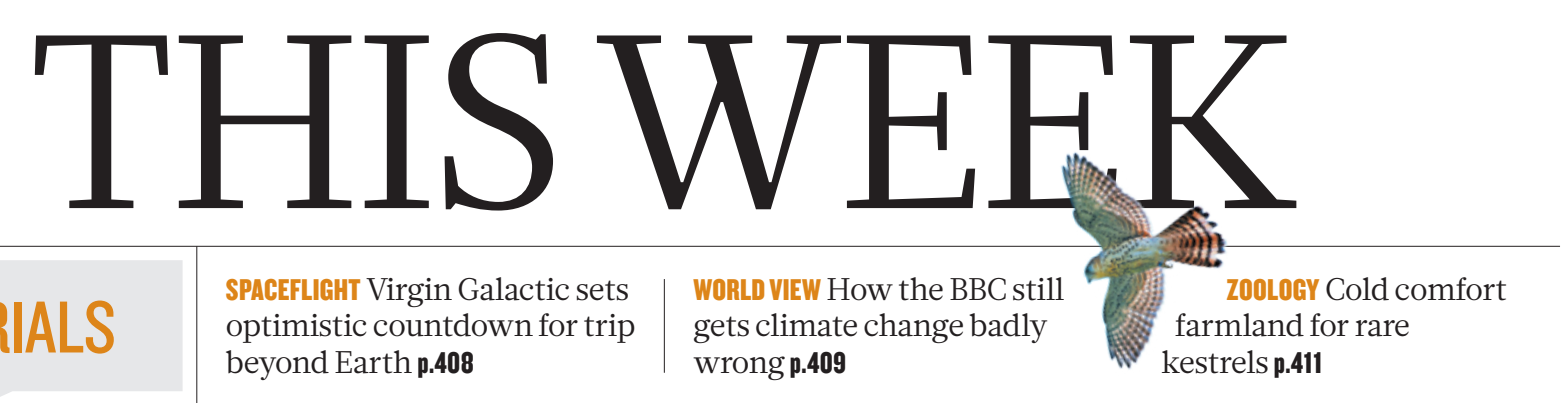

EDITORIALS optimistic countdown for trip beyond Earth $\mathbf{p . 4 0 8}$

\title{
Fish have feelings too
}

\author{
Our obligation to keep the suffering of laboratory animals to a minimum - both in life and in \\ death - does not apply only to mammals.
}

$\mathrm{F}$ Tormer US President George W. Bush once cryptically remarked that he was sure that "the human being and fish can coexist peacefully". Fish might beg to differ. Humans continue to deplete ocean stocks for food and to stalk rivers in the name of sport. And then there are the millions of fish that die in scientific laboratories every year.

Much of the debate about the use of animals in research focuses on what happens while they are alive: the degree of pain and suffering inflicted on them, how this can be kept to a minimum, and the balance between this discomfort and the greater benefit it can and does bring to both people and animals. Less talked about - perhaps fortuitously, given the way emotion can drive such debates - is the fact that most of the animals lose more than their freedom and their comfort. The majority of laboratory animals are killed at the end of the work. Killing animals is an unpleasant thing to have to do, but unfortunately in some areas of science it is unavoidable. So it is important that the regulations scientists follow for animal euthanasia reflect the most humane options available. New research suggests that this might not always be the case for zebrafish.

A native of the southeastern Himalayas, zebrafish are one of the most commonly used model organisms in research. One reason for their popularity is that zebrafish embryos are transparent, making them an ideal organism in which to track development, as well as the influence that genes and other factors have on this process. As tools to exploit this unusual embryonic feature have grown, so has the use of zebrafish. Exact numbers are hard to find, but the total number of fish used in UK laboratories is second only to the number of mice.

Another factor that makes zebrafish attractive is that the creatures are relatively cheap to house. They need less space, less dedicated staff time and there are fewer regulations than for rodents on, say, habitat enrichment.

In the hierarchy of living things, fish are viewed by many as a lesser life form than mammals, but not everyone agrees. A small but committed number of animal-rights campaigners, for example, extend their attention to fish and related creatures. Certainly, whether hooked fish feel pain and what, if anything, lobsters experience when dropped live into boiling water are genuine scientific questions, and ones with the potential to stir feelings and change behaviour.

The questions raised this week concern the ways in which zebrafish used in laboratories are killed at the end of their useful lives. As we report on page 419, experimental work suggests that an approved anaesthetic commonly used in fish euthanasia causes the creatures distress.

Many researchers will rightly respond cautiously to calls to change regulations and long-standing experimental practice on the basis of just two papers, which are detailed in the News story. And it is not clear whether alternative options to the anaesthetic, MS-222, are more humane.
As animal-welfare researchers have probed the methods used in laboratory euthanasia, they have provoked sometimes fierce debate over which is the kindest option. The widespread use of carbon dioxide in rodent euthanasia has come under particular scrutiny, because
"It is important

that the

regulations

scientists follow

for animal

euthanasia

reflect the most

humane options available." evidence is growing that this method is more unpleasant for rats and mice than alternatives such as anaesthesia.

At the very least, the work on the zebrafish should prompt reassessment of how much we know about some routine practices of animal research. Frequently, when researchers have probed the assumptions underpinning laboratory standards they have exposed flaws. And fixing these flaws - such as that revealed by the finding that rodents should not be picked up by their tails because this causes them stress, which could interfere with the results of some experiments - can produce better science.

Most laboratory animals are looked after by skilled and dedicated technicians and scientists who care deeply about the creatures' welfare. Their existence may not always be peaceful, but we can - and must — try to make it so, especially at the end.

\section{Parallel lines}

\section{A collaborative online mathematics project holds lessons for other disciplines.}

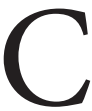

rowd-sourcing has reached mathematics, and at first glance it might seem as if this stereotypically solitary discipline is finally catching up with what other sciences have been doing for years. But, as we explore on page 422 , the maths project Polymath, which invites participants to pitch in with ideas and results that might help to solve whatever problem the coordinator has set, is in some ways ahead of the curve. Not all of Polymath's challenges - nine so far - have produced a successful solution. But even 'failures' can be productive, and all of these efforts represent genuine collaborations at the highest technical level.

It is in these respects that Polymath differs from the many other crowd-sourcing enterprises. Most commercial ventures are competitive: entrants vie to 'win' the challenge, and often to receive a financial prize as a result. As one researcher who has used these resources comments, this isn't necessarily the way to secure a truly useful solution: an extended period of post-competition development is often needed 